PSICOLOGIA

IBEROAMERICANA
Psicología Iberoamericana ISSN: 1405-0943

revista.psicologia@ibero.mx

Universidad Iberoamericana, Ciudad de México México

\title{
Implicaciones del sistema serotoninérgico y la neuroglia en lo mecanismos del estrés: Una breve revisión
}

González Rivera, Ivette; Paz Trejo, Diana Berenice; Galicia Castillo, Oscar; Sánchez Castillo, Hugo Implicaciones del sistema serotoninérgico y la neuroglia en lo mecanismos del estrés: Una breve revisión

Psicología Iberoamericana, vol. 26, núm. 1, 2018

Universidad Iberoamericana, Ciudad de México, México

Disponible en: http://www.redalyc.org/articulo.oa?id=133959553004 


\title{
Implicaciones del sistema serotoninérgico y la neuroglia en lo mecanismos del estrés: Una breve revisión
}

\author{
Implications of the serotonergic system and neuroglia in the \\ mechanisms of stress: A short review \\ Ivette González Rivera igr.psi@gmail.com \\ Universidad Nacional Autònoma de Mèxico , México \\ Diana Berenice Paz Trejo \\ Sociedad Iberoamericana de Neurociencia Aplicada, México \\ Oscar Galicia Castillo \\ Universidad Iberoamericana, México \\ Hugo Sánchez Castillo \\ Universidad Nacional Autónoma de México, México
}

Resumen: La respuesta de estrés puede variar dependiendo de la intensidad del estresor, así como de factores inherentes al organismo. De manera general, cuando un sujeto desarrolla trastornos relacionados con el estrés, ocurren cambios anatómicos y fisiológicos en el sistema nervioso central ligados a los síntomas que se presentan. Comúnmente se usan fármacos serotoninérgicos como tratamiento para dichos síntomas; sin embargo, se desconoce la acción precisa de éstos sobre la actividad neural y se conoce mucho menos sobre la participación de la neuroglia en este proceso. El presente artículo muestra la relevancia del sistema serotoninérgico en la respuesta de estrés, así como la posible participación de la neuroglia, particularmente de los astrocitos, como una clave para entender mejor los mecanismos del estrés y la recuperación del sistema por vías serotoninérgicas particulares.

Palabras clave: astrocitos, estrés, neuroglia, receptores 5 HT1A, sistema serotoninérgico. Abstract: The stress response may vary depending on the intensity of the stressor, as well as factors inherent to the organism. When the subject develops stress-related disorders, anatomical and physiological changes occur in the central nervous system linked to the symptoms. Serotonergic drugs are commonly used as a treatment for such symptoms. However, their precise action on neural activity is unknown, and less is known about the involvement of neuroglia in this process. The present article demonstrates the relevance of the serotoninergic system in the stress response and the possible participation of the neuroglia, particularly astrocytes, as a key to understanding the mechanisms of stress and the recovery of the system by particular serotonergic pathways.

Keywords: astrocytes, neuroglia, 5HT1A receptors, stress, serotonergic system.

\section{INTRODUCCIÓN}

La respuesta de los organismos ante situaciones estresantes ha sido un tema importante de estudio en la investigación debido a la relevancia que supone este mecanismo para la supervivencia. En múltiples estudios se ha encontrado que la exposición a estrés implica la activación de diversos sistemas de NeuroModulación, tales como la norepinefrina, serotonina (5-HT), dopamina, gaba y endocannabinoides (Miguel-Tobal, González, \& López, 2000). El sistema serotoninérgico es uno de los más claramente 
involucrados en la respuesta de estrés, sin embargo, se desconoce su acción precisa y el mecanismo por el cual fármacos serotoninérgicos ejercen un efecto terapéutico en trastornos asociados al estrés, como la ansiedad y la depresión.

Asimismo, con la investigación se ha ido dejando atrás la visión neurocéntrica de los procesos centrales, dando lugar al estudio del rol de las células gliales en los mecanismos del estrés. Particularmente, se ha encontrado que los astrocitos juegan un papel importante en la respuesta normal a una situación estresante y que también se ven afectados en las patologías relacionadas con el estrés (Bender, Calfa, \& Molina, 2016). Sin embargo, aún permanece desconocido cómo el sistema serotoninérgico, en conjunto con estas células, interactúa para generar la respuesta normal y patológica ante los distintos tipos de estrés.

En ese sentido, resulta relevante contar con información sobre el curso actual de la investigación en este campo, así como las rutas posibles de investigación en las que el cruce del sistema serotoninérgico y las células gliales puedan ofrecer una clave para entender mejor los mecanismos de respuesta al estrés. El presente artículo constituye una breve revisión enfocada a estas dos variables en conjunto, con el objetivo de identificar elementos que permitan entender mejor el rol de la serotonina y los astrocitos en el estrés, así como de la relevancia de este tema en la investigación de la terapéutica actual.

\section{La Respuesta de Estrés}

Históricamente, el término estrés ha tenido una connotación de tensión, presión y enfrentamiento de adversidades; sin embargo, esta definición se ha modificado a través del tiempo (Molina-Jiménez, Gutiérrez- García, Hernández-Domínguez, \& Contreras, 2008).

El concepto de estresor se refiere a una situación que es percibida como peligrosa y perturba la homeostasis del organismo (Molina-Jiménez et al., 2008), mientras que el concepto estrés denota la relación que existe entre dichos estímulos estresantes y las respuestas fisiológicas y conductuales del organismo ante esta estimulación (Bruner \& Vargas, 1994).

Una de las primeras respuestas ante las experiencias estresantes es la activación del sistema nervioso autónomo, que resulta en una rápida liberación de adrenalina, así como en la activación del sistema cardiovascular, respiratorio y metabólico (Molina- Jiménez et al., 2008). Asimismo, ocurre una activación de estructuras a nivel del sistema nervioso central (snc), tales como la liberación de noradrenalina en las proyecciones noradrenérgicas que surgen desde el locus coeruleus (lc) (Roozendaal, McEwen, \& Chattarji, 2009) y la activación del eje hipotálamo-pituitario-adrenal (hpa) (De Kloet, Joëls, \& Holsboer, 2005).

Este proceso de activación fisiológico también impacta a nivel conductual y cognitivo, y normalmente se detiene cuando el estímulo estresante cesa, de manera que se trata de un proceso de adaptación al entorno que permite la supervivencia (Carrasco \& Van de Kar, 2003; De 
Kloet et al., 2005). En ese sentido, se han propuesto dos tipos de respuestas al estrés, llamadas eustrés y distrés. El eustrés se considera una respuesta adaptativa que permite afrontar los estímulos estresantes de manera exitosa, y que permite la funcionalidad del sujeto, ya que el mecanismo de activación fisiológica se detiene cuando deja de ser necesario. Por su parte, el distrés se considera una respuesta del organismo que no genera ningún beneficio en el funcionamiento del sujeto, por lo que culmina en deterioro de su bienestar (Cano-Vindel, 2003; Molina-Jiménez et al., 2008).

Asimismo, ante la exposición a eventos estresantes que pongan en riesgo la integridad del individuo, no solamente se afecta la cualidad de la respuesta, sino también su permanencia en el tiempo. De esta manera, pueden llegar a presentarse diversos trastornos relacionados con el estrés, entre los cuales se encuentran los de ansiedad y del estado de ánimo, tales como la ansiedad generalizada y depresión mayor (Sánchez, Paz-Trejo, Vazquéz, Zarate, \& Migliaro, 2014).

\section{Mecanismo Central del Estrés}

La respuesta de estrés a nivel del snc puede variar dependiendo de la intensidad del estresor, así como de factores inherentes al organismo que pueden predisponerlo a desarrollar un trastorno. De manera general, cuando el sujeto desarrolla trastornos relacionados con el estrés, ocurren cambios anatómicos y fisiológicos que perduran en el sistema y que se han ligado a los síntomas que se presentan en dichas patologías (Sánchez et al., 2014).

Una de las estructuras más afectadas es el hipocampo, dada su alta concentración de receptores a glucocorticoides y su sensibilidad a cambiosx por el ambiente y la experiencia (Gould \& Tanapat, 1999). Diversos estudios muestran una reducción del volumen del hipocampo y la pérdida de neuronas y espinas dendríticas después de la exposición a niveles altos de glucocorticoides (Sapolsky, 1996), así como la disminución de neuroglia en el hipocampo y otras estructuras límbicas ante la exposición a estrés crónico (Rajkowska \& Miguel-Hidalgo, 2007).

Por su parte, la amígdala es de relevancia para el procesamiento de las emociones, así como para la puesta en marcha de las respuestas de ansiedad y miedo (LeDoux, 2000; Wilson \& Rolls, 1990). De hecho, la falla en la extinción del miedo se relaciona con alteraciones en la amígdala, así como con una conexión deficiente entre las proyecciones de la corteza prefrontal medial (cpfm) y la amígdala (Sánchez et al., 2014; Tovote, Fadok, \& Lüthi, 2015).

De manera similar, se ha encontrado que pacientes con trastornos relacionados con el estrés tienen una disminución de la actividad de la cpfm y presentan alteraciones en la memoria de trabajo y la regulación atencional (Bremner et al., 1997; Bremner et al., 1999); esto es particularmente relevante puesto que la cpfm interviene en la modulación del balance emocional asociado a memorias específicas y en la inhibición de la respuesta de la amígdala (Ochsner et al., 2004), cuya disfunción se hace evidente en la sintomatología de estos trastornos. 


\section{Sistema Serotoninérgico y Estrés}

Una de las primeras respuestas al estrés es un incremento de la serotonina en la amígdala desde los núcleos dorsales del rafé. De hecho, la excitabilidad neuronal y la transmisión sináptica en la amígdala se modulan por la activación de receptores de serotonina durante las experiencias estresantes (Amat, Matus-Amat, Watkins, \& Maier, 1998; Minor \& Hunter, 2002). La acción primaria del incremento de serotonina es la reducción de la excitabilidad de la amígdala por un incremento de la transmisión gabaérgica en el núcleo basolateral de la amígdala (Jiang et al., 2008; Rainnie, 1999). Debido a que la modulación serotoninérgica en la amígdala es de gran importancia en la normalización del procesamiento de las señales emocionales y del estrés, su desregulación puede derivar en respuestas anormales a los estímulos emocionales, ansiedad y depresión (Hariri et al., 2002).

Asimismo, existen importantes interacciones recíprocas entre los sistemas noradrenérgico y serotoninérgico. El disparo de las neuronas noradrenérgicas en el lc está bajo la inhibición tónica de proyecciones desde el núcleo dorsal del rafé; de esta manera el uso de inhibidores selectivos de la recaptura de serotonina puede incidir indirectamente en la inhibición del tono noradrenérgico (Szabo \& Blier, 2001). La serotonina también modula efectos en el eje hpa. Se ha propuesto que puede actuar para inhibir el nacimiento de la hormona liberadora de corticotropina en el núcleo paraventricular directa o indirectamente (Kent, Coplan, \& Gorman, 1998).

Finalmente, síntomas asociados al estrés (como irritabilidad, impulsividad, alteraciones en el consumo de alimentos, desregulación en el sueño y cambios en la actividad cardiovascular y respiratoria), así como trastornos relacionados con el estrés (como la depresión y la ansiedad generalizada), están relacionados con la modulación serotoninérgica. Asimismo, en el campo clínico se han usado diversos agentes farmacológicos serotoninérgicos para el tratamiento de pacientes con trastornos relacionados al estrés, incluyendo inhibidores selectivos de la recaptura de serotonina (isrs), inhibidores de monoamina oxidasa (imao) y antidepresivos tricíclios (Daskalakis, Yehuda, \& Diamond, 2013). La mejora de los síntomas, seguida por el tratamiento con antidepresivos, también sostiene el rol de importancia del sistema serotoninérgico en dichos trastornos (Evenden, 1999; Monti \& Jantos, 2008; Villalón \& Centurión, 2007).

Sin embargo, la vía por la cual los fármacos serotoninérgicos regulan diversas alteraciones no está del todo clara. Por ejemplo, en un estudio en el que compararon los efectos de fármacos isrs y tricíclicos sobre el factor de crecimiento neural y el factor de crecimiento endotelial vascular, se encontraron efectos regulatorios con la administración de isrs, pero no con la administración de tricíclicos. Esto podría indicar que la expresión de factores neurotróficos puede estar mediada por mecanismos específicos de los isrs, pero se desconoce cuáles particularmente (Allaman, Fiumelli, Magistretti, \& Martin, 2011), lo que indica que se requiere un estudio 
más profundo de los factores particulares por los cuales lo fármacos serotoninérgicos ejercen sus efectos regulatorios.

De esta manera, surge la necesidad de buscar fármacos más selectivos que brinden un tratamiento más eficaz y que permitan profundizar en el conocimiento de los receptores serotoninérgicos particulares afectados en el estrés. Esto debido a que los receptores serotoninérgicos participan en múltiples funciones fisiológicas, psicoafectivas y en condiciones patológicas, entre las cuales se encuentran algunas muy ligadas a los procesos de respuesta al estrés. Por ejemplo, los receptores 5-HT1A y 5-HT2C están implicados en trastornos de ansiedad, depresión y en la regulación de la conducta sexual; los receptores 5-HT1D y 5-HT1B participan en la regulación del consumo de alimentos; los receptores 5HT2A regulan los procesos de sueño-vigilia; y los receptores 5-HT3-7 se han asociado a procesos cognitivos (Lanfumey, Mongeau, CohenSalmon, \& Hamon, 2008). Es por ello que receptores particulares del sistema serotoninérgico se han sugerido como posibles objetivos farmacológicos más específicos.

En ese sentido, estudios con animales sugieren que la disminución en la señalización del receptor 5-HT2A está relacionada con la ocurrencia de los síntomas psiquiátricos asociados al estrés (Dwivedi, Mondal, Payappagoudar, \& Rizavi, 2005; Wu et al., 1999). Después de la administración de antagonistas del receptor 5-HT2 se encuentra mejora en algunos de los síntomas de pacientes con Trastorno de Estrés Postraumático, particularmente con la nefazodona (Davis et al., 2004; Garfield, Fichtner, Leveroni, \& Mahableshwarkar, 2001; Gillin et al., 2001). Del mismo modo, los receptores 5-HT1A son una importante diana terapéutica debido a su alta concentración en la corteza prefrontal y en regiones involucradas en el control de las emociones, de la cognición y de la memoria. Los receptores presinápticos 5-HT1A se encuentran principalmente en las células de los núcleos del Rafé, mientras que los receptores postsinápticos se encuentran en el hipocampo, septum, amígdala, corteza entorrinal y corteza cerebral (Newman-Tancredi, 2011). Además, hay abundantes receptores 5-HT1A en astrocitos y otras células gliales (Kettenmann \& Ransom, 2013).

Asimismo, los receptores 5-HT1A se han relacionado con los mecanismos de acción de ansiolíticos, anti-depresivos y antipsicóticos; de hecho, la mayoría de los antidepresivos (imao, atc, isrs, litio, valproato) aumenta la señalización postsináptica de los receptores 5-HT1A, ya sea por mecanismos directos o indirectos (Savitz, Lucki, \& Drevets, 2009). La participación de estos receptores particulares en trastornos de ansiedad y del estado de ánimo es cada vez más clara. Ejemplo de esto es un estudio en el que se encontró un aumento de las conductas de ansiedad en ratones knockout de receptores 5-HT1A (Parks, Robinson, Sibille, Shenk, \& Toth 1998). Otros estudios han relacionado el trastorno de pánico con una disfunción en los receptores 5-HT1A (Nash et al., 2008; Neumeister et al., 2004) y han encontrado una reducción de receptores 5-HT1A en pacientes con depresión mayor (Savitz et al., 2009). Asimismo, existe evidencia de que la serotonina puede estimular la producción de neuronas 
en el giro dentado del hipocampo a través de los receptores 5-HT1A (Gould, 1999; Mahar, Bambico, Mechawar, \& Nobrega, 2014).

Esta evidencia propone fuertemente que los receptores 5-HT1A son un objetivo de investigación importante para el tratamiento de trastornos relacionados con el estrés, así como para otros desórdenes neuropsiquiátricos (Newman-Tancredi \& Kleven, 2011).

\section{Sistema Serotoninérgico y Neuroglia}

Las células gliales tienen una importante participación en la mayoría de los procesos cerebrales; de hecho, algunos hallazgos sugieren que estas células podrían ser clave para mejorar nuestro conocimiento del snc y resolver algunos problemas que permanecen en la neurobiología de diversas alteraciones (Jauregui-Huerta et al., 2010).

Los trastornos relacionados con el estrés no son la excepción, diversos estudios han encontrado alteraciones en la neuroglia en modelos animales y en pacientes después de la exposición a estrés crónico o agudo (Bender et al., 2016). Por ejemplo, en pacientes con depresión mayor se ha encontrado una reducción en neuroglia en regiones fronto-límbicas, así como una reducción de la densidad de la oligodendroglia y alteraciones en la expresión genética de factores neurotróficos de transportadores gliales (Rajkowska \& Miguel-Hidalgo, 2007).

Particularmente, se ha encontrado que los astrocitos son una de las células gliales más involucradas en las patologías de estrés y sobreproducción de glucocorticoides. De hecho, la pérdida de volumen en el hipocampo y las alteraciones en la corteza prefrontal pueden estar relacionadas con una reducción de astroglia. En modelos animales, la exposición a estrés crónico resulta en la disminución de gliogénesis en estructuras límbicas y en la corteza prefrontal (Czéh, Simon, Schmelting, Hiemke, \& Fuchs, 2006) así como en una reducción de células gliales, incluso mayor que de neuronas, después de exposición prolongada a esteroides (Rajkowska \& Miguel-Hidalgo, 2007).

De hecho, las ratas expuestas a estrés crónico impredecible muestran déficits en conducta en el test de nado forzado y muestran una disminución significativa de la proteína ácida fibrilar glial en el hipocampo; también muestran una mejora con el tratamiento de clomipramina, un antidepresivo tricíclico, lo que sustenta la hipótesis de que la disfunción glial contribuye a la patofisiología de la depresión y el estrés, y que la recuperación por antidepresivos puede estar mediada por estas células (Liu et al., 2009).

Banasr y Duman (2008) encontraron que la ablación de glía en la corteza prefrontal es suficiente para inducir conducta depresiva similar a la generada por estrés, mientras que Hamidi, Drevets y Price (2004) encontraron que la reducción en el volumen amigdalino en pacientes con trastorno depresivo se debe principalmente a una reducción de oligodendrocitos.

Por su parte, Leventopoulos et al. (2007) encontraron que la exposición temprana a estrés genera cambios a largo plazo en la densidad de astroglia 
en regiones del cerebro involucrados en la respuesta al estrés, tales como el giro dentado dorsal, la corteza prefrontal, el hipocampo ventral y dorsal, el área CA1, el hipocampo basolateral, la corteza cingulada y la amígdala.

Finalmente, Czéh et al. (2006) sometieron a un protocolo de estrés psicosocial a musarañas arborícolas y encontraron una reducción de $25 \%$ tanto en el número como en el volumen de la astroglía en el hipocampo. De esta manera, sugieren que la reducción en los astrocitos puede estar altamente relacionada con efectos de toxicidad glutaminérgica en las neuronas y sobre factores neurotróficos. Asimismo, el tratamiento con fluoxetina previo al estrés ofreció protección a la disminución del número de astrocitos. Dicha protección puede estar estrechamente relacionada con el hecho de que los astrocitos pueden captar serotonina por canales dependientes de sodio y además expresan muchos subtipos de receptores serotoninérgicos tales como el 5-HT1A y 5-HT2A (Azmitia, Gannon, Kheck, \& Whitaker-Azmitia, 1996).

Esta evidencia muestra que la participación de los astrocitos en el estrés está ligada de manera importante al sistema serotoninérgico, y que los efectos de los fármacos serotoninérgicos sobre estas células pueden ser una vía por la cual ejercen sus efectos terapéuticos cuando son administrados en pacientes con trastornos relacionados con el estrés, o en modelos animales de los mismos.

\section{CONCLUSIONES}

El sistema serotoninérgico se encuentra altamente implicado tanto en el eustrés como en el distrés, esto indica que este sistema de neurotransmisión es clave para entender mejor la respuesta de los organismos a los eventos estresantes, así como las patologías asociadas a su exposición prolongada. Particularmente, los resultados de diversas investigaciones (Mahar et al., 2014; Nash et al., 2008; Neumeister et al., 2004; Savitz et al., 2009) muestran que los receptores 5HT1A son un blanco importante para generar tratamientos más efectivos, y que su complejo efecto tanto a nivel presináptico como postsináptico puede brindar más información de la neurobiología de la respuesta al estrés.

Asimismo, diversos estudios (Banasr \& Duman, 2008; Czéh et al., 2006; Leventopoulos et al., 2007; Liu et al., 2009) indican que las células gliales, particularmente los astrocitos, tienen un papel importante en la respuesta al estrés, ya que estas células se han encontrado alteradas después de la exposición a eventos estresantes. De manera interesante, estas alteraciones son reguladas con la administración de fármacos serotoninérgicos, lo que sugiere que los síntomas asociados a los trastornos relacionados con el estrés pueden estar ligados con cambios en la anatomía de los astrocitos y que el efecto terapéutico de los fármacos serotoninérgicos podría estar a su vez asociado con estas células, por lo que la relación estrésastrocitosserotonina resulta ser un campo de investigación muy atractivo.

Es claro que se requiere más información sobre el papel de las células gliales en los trastornos relacionados con el estrés, así como sobre su 
relación con receptores particulares del sistema serotoninérgico para comprender mejor el efecto del estrés en el snc y su impacto en la cognición y la conducta. Igualmente, es necesario profundizar en el mecanismo de acción particular por el cual los fármacos antidepresivos ejercen su efecto sobre la sintomatología del estrés, posiblemente con el uso de fármacos específicos a ciertos receptores serotoninérgicos que brinden información que permita ampliar las posibilidades de tratamientos farmacológicos y que contribuya a entender mejor los mecanismos de los trastornos relacionados con el estrés y su recuperación.

\section{REFERENCIAS}

Allaman, I., Fiumelli, H., Magistretti, P., \& Martin, J. L. (2011). Fluoxetine regulates the expression of neurotrophic/growth factors and glucose metabolism in astrocytes. Psychopharmacology, 216(1), 75-84. https://do i.org/10.1007/s00213-011-2190-y

Amat, J., Matus-Amat, P., Watkins, L. R., \& Maier, S. F. (1998). Escapable and inescapable stress differentially alter extracellular levels of 5-HT in the basolateral amygdale of the rat. Brain Research, 812, 113-120. https://do i.org/10.1016/S0006-8993(98)00960-3

Azmitia, E. C. Gannon, P.J., Kheck, N. M., \& Whitaker-Azmitia, P. M. (1996). Cellular localization of the 5-HT1A receptor in primate brain neurons and glial cells. Neuropsychopharmacology, 14(1), 35- 46.

Banasr, M., \& Duman, R.S. (2008). Glial loss in the prefrontal cortex is sufficient to induce depressive-like behaviors. Biological Psychiatry, 64(10), 863-870. https://doi.org/10.1016/j.biopsych.2008.06.008

Bender, C. L., Calfa, G. D., \& Molina, V. A. (2016). Astrocyte plasticity induced by emotional stress: A new partner in psychiatric physiopathology? Progress in Neuro-Psychopharmacology and Biological Psychiatry, 65(4), 68-77. https://doi.org/10.1016/j.pnpbp.2015.08.005

Bremner, J. D., Innis, R. B., Ng, C. K., Staib, L. H., Salomon, R. M., Bronen, R. A., Duncan, J., Southwick S. M., Krystal, J. H., Rich, D., Zubal, G., Dey, H. Soufer, R., \& Charney, D. S. (1997). Positron emission tomography measurement of cerebral metabolic correlates of yohimbine administration in combat-related post-traumatic stress disorder. Archives of General Psychiatry, 54(3), 246-254. https://doi.org/10.1001/archpsyc .1997 .01830150070011

Bremner, J. D., Narayan, M., Staib, L. H., Southwick, S. M., McGlashan, T., \& Charney, D. S. (1999). Neural correlates of memories of childhood sexual abuse in women with and without post- traumatic stress disorder. The American Journal of Psychiatry, 156(11), 1787-1795.

Bruner, C. A., \& Vargas, I. (1994). The activity of rats in a swimming situation as a function of water temperature. Physiology and Behavior, 55(1), 21-28. https://doi.org/10.1016/0031-9384(94)90004-3

Cano-Vindel, A. (2003). Desarrollos actuales en el estudio del control emocional. Ansiedad y Estrés, 9(2-3), 203-229.

Carrasco, G. A., \& Van de Kar, L. D. (2003). Neuroendocrine pharmacology of stress. European Journal of Pharmacology, 463(1-3), 235-272. https://doi .org/10.1016/S0014-2999(03)01285-8 
Czéh, B., Simon, M., Schmelting, B., Hiemke, C., \& Fuchs, E. (2006). Astroglial plasticity in the hippocampus is affected by chronic psychosocial stress and concomitant fluoxetine treatment. Neuropsychopharmacology, 31(8), 1616-1626.

Daskalakis, N. P., Yehuda, R., \& Diamond, D. M. (2013). Animal models in translational studies of PTSD. Psychoneuroendocrinology, 38(9), 1895-1911. https://doi.org/10.1016/j.psyneuen.2013.06.006

Davis, L. L., Jewell, M. E., Ambrose, S., Farley, J., English, B., Bartolucci, A., \& Petty, F. (2004). A placebo-controlled study of nefazodone for the treatment of chronic posttraumatic stress disorder: A preliminary study. Journal of Clinical Psychopharmacology, 24(3), 291-297. https://doi.org/ 10.1097/01.jcp.0000125685.82219.1a

De Kloet, E. R., Joëls, M., \& Holsboer, F. (2005). Stress and the brain: From adaptation to disease. Nature Reviews Neuroscience, 6(6), 463- 475.

Dwivedi, Y., Mondal, A. C., Payappagoudar, G. V., \& Rizavi, H. S. (2005). Differential regulation of serotonin $(5-\mathrm{HT}) 2 \mathrm{~A}$ receptor mRNA and protein levels after single and repeated stress in rat brain: Role in learned helplessness behavior. Neuropharmacology, 48(2), 204-214. https://doi.o $\mathrm{rg} / 10.1016 /$ j.neuropharm.2004.10.004

Evenden, J. (1999). Impulsivity: A discussion of clinical and experimental findings. Journal of Psychopharmacology, 13(2), 180-192. https://doi.org/ 10.1177/026988119901300211

Garfield, D. A., Fichtner, C. G., Leveroni, C., \& Mahableshwarkar, A. (2001). Open trial of nefazodone for combat veterans with posttraumatic stress disorder. Journal of Traumatic Stress, 14(3), 453-460. https://doi.org/10 .1023/A:1011148304140

Gillin, J. C., Smith-Vaniz, A., Schnierow, B., Rapaport, M. H., Kelsoe, J., Raimo, E. M., Matthew, R., Goyette, L. M., Stein, M., \& Zisook, S. (2001). An open-label, 12-week clinical and sleep eeg study of nefazodone in chronic combat-related posttraumatic stress disorder. Journal of Clinical Psychiatry, 62(10), 789-796. http://dx.doi.org/10.4088/JCP.v62n1007

Gould, E. (1999). Serotonin and hippocampal neurogenesis. Neuropsychopharmacology, 21, 46S-51S.

Gould, E., \& Tanapat, P. (1999). Stress and hippocampal neurogenesis. Biological Psychiatry, 46(11), 1472-1479. https://doi.org/10.1016/S000 6-3223(99)00247-4

Hamidi, M., Drevets, W. C., \& Price, J. L. (2004). Glial reduction in amygdala in major depressive disorder is due to oligodendrocytes. Biological Psychiatry, 55(6), 563-569. https://doi.org/10.1016/j.biopsych.2003.11.006

Hariri, A. R., Mattay, V. S., Tessitore, A., Kolachana, B., Fera, F., Goldman, D., Egan, M. F., \& Weinberger, D. R. (2002). Serotonin transporter genetic variation and the response of the human amygdala. Science, 297(5580), 400-403. https://doi.org/10.1126/science.1071829

Jauregui-Huerta, F., Ruvalcaba-Delgadillo, Y., Gonzalez-Castañeda, R., GarciaEstrada, J., Gonzalez-Perez, O., \& Luquin, S. (2010). Responses of glial cells to Stress and Glucocorticoids. Current Immunology Reviews, 6(3), 195-204. https://doi.org/10.2174/157339510791823790

Jiang, X., Xing, G., Yang, C., Verma, A., Zhang, L., \& Li, H. (2008). Stress impairs 5-HT2A receptor-mediated serotonergic facilitation of GABA 
release in juvenile rat basolateral amygdala. Neuropsychopharmacology, 34(2), 410-423.

Kent, J. M., Coplan, J. D., \& Gorman, J. M. (1998). Clinical utility of the selective serotonin reuptake inhibitors in the spectrum of anxiety. Biological Psychiatry, 44(9), 812-824. https://doi.org/10.1016/S0006-32 23(98)00210-8

Kettenmann, H., \& Ransom, B. R. (2013). Neuroglia. Oxford, UK: Oxford University Press.

Lanfumey, L., Mongeau, R., Cohen-salmon, C., \& Hamon, M. (2008). Corticosteroid-serotonin interactions in the neurobiological mechanisms of stress-related disorders. Neuroscience and Biobehavioral Reviews, 32(6), 1174-1184. https://doi.org/10.1016/j.neubiorev.2008.04.006

LeDoux, J. E. (2000). Emotion circuits in the brain. Annual Review of Neuroscience, 23, 155-184. https://doi.org/10.1146/annurev.neuro.23.1. 155

Leventopoulos, M., Rüedi-Bettschen, D., Knuesel, I., Feldon, J., Pryce, C. R., \& Opacka-Juffry, J. (2007). Long-term effects of early life deprivation on brain glia in Fischer rats. Brain Research, 1142, 119-126. https://doi.org/ 10.1016/j.brainres.2007.01.039

Liu, Q., LI, B., Zhu, H. Y., Wang, Y. Q., Yu, J., \& Wu, G. C. (2009). Clomipramine treatment reversed the glial pathology in a chronic unpredictable stress-induced rat model of depression. European Neuropsychopharmacology, 19(11), 796-805. https://doi.org/10.1016/j.e uroneuro.2009.06.010

Mahar, I., Bambico, F. R., Mechawar, N., \& Nobrega, J. N. (2014). Stress, serotonin, and hippocampal neurogenesis in relation to depression and antidepressant effects. Neuroscience \& Biobehavioral Reviews, 38, 173-192. https://doi.org/10.1016/j.neubiorev.2013.11.009

Miguel-Tobal, J. J., González, H., \& López, E. (2000). Estrés postraumático: Hacia una integración de aspectos psicológicos y neurobiológicos. Ansiedad y Estrés, 6(2-3), 255-280.

Minor, T. R., \& Hunter, A. M. (2002). Stressor controllability and learned helplessness research in the United States: Sensitization and fatigue processes. Integrative Physiological and Behavioral Science, 37(1), 44-58. h ttps://doi.org/10.1007/BF02688805

Molina-Jiménez, T., Gutiérrez-García, A. G., Hernández-Domínguez, L., \& Contreras, C. M. (2008). Estrés psicosocial: Algunos aspectos clínicos y experimentales. Anales de Psicología, 24(2), 353-360.

Monti, J. M., \& Jantos, H. (2008). The roles of dopamine and serotonin, and of their receptors, in regulating sleep and waking. Progress in Brain Research, 172, 625-646. https://doi.org/10.1016/S0079-6123(08)00929-1

Nash, J. R., Sargent, P. A., Rabiner, E. A., Hood, S. D., Argyropoulos, S. V., Potokar, J. P., Grasby, P. M., \& Nutt, D. J. (2008). Serotonin 5HT1A receptor binding in people with panic disorder: Positron emission tomography study. British Journal of Psychiatry, 193(3), 229-234. https:/ /doi.org/10.1192/bjp.bp.107.041186

Neumeister, A., Bain, E., Nugent, A. C., Carson, R. E., Bonne, O., Luckenbaugh, D. A., Eckelman, W., Herscovitch, P., Charney, D. S., \& Drevets, W. C (2004). Reduced serotonin type 1A receptor binding in panic disorder. 
The Journal of Neuroscience, 24(3), 589-591. https://doi.org/10.1523/JN EUROSCI.4921-03.2004

Newman-Tancredi, A. (2011). Biased agonism at serotonin 5-HT1 A receptors: Preferential postsynaptic activity for improved therapy of CNS disorders. Neuropsychiatry, 1(2), 149-164. https://doi.org/10.2217/NPY.11.12

Newman-Tancredi, A., \& Kleven, M. S. (2011). Comparative pharmacology of antipsychotics possessing combined dopamine D2 and serotonin 5HT1A receptor properties. Psychopharmacology, 216(4), 451-473. https: //doi.org/10.1007/s00213-011-2247-y

Ochsner, K. N., Ray, R. D., Cooper, J. C., Robertson, E. R., Chopra, S., Gabriele, J. D. E., \& Gross, J. J. (2004). For better or for worse: Neural systems supporting the cognitive down-and up-regulation of negative emotion. Neuroimage, 23(2), 483-499. https://doi.org/10.1016/j.neuroimage.200 4.06.030

Parks, C. L., Robinson, P. S., Sibille, E., Shenk, T., \& Toth, M. (1998). Increased anxiety of mice lacking the serotonin $1 \mathrm{~A}$ receptor. Proceedings of the National Academy of Sciences of the United States of America, 95(18), 10734-10739.

Rainnie, D. G. (1999). Serotonergic modulation of neurotransmission in the rat basolateral amygdala. Journal of Neurophysiology, 82(1), 69-85. https://d oi.org/10.1152/jn.1999.82.1.69

Rajkowska, G., \& Miguel-Hidalgo, J. J. (2007). Gliogenesis and glial pathology in depression. CNS \& Neurological Disorders Drug Targets, 6(3), 219-233. https://doi.org/10.2174/187152707780619326

Roozendaal, B., McEwen, B. S., \& Chattarji, S. (2009). Stress, memory and the amygdala. Nature Reviews Neuroscience, 10(6), 423-433.

Sánchez, H., Paz-Trejo, D., Vazquéz, J., Zarate, P., \& Migliaro, M. (2014). Neurobiology of posttraumatic stress disorder (ptsd) and its frontostriatal implications: A short review. Actualidades en Psicologia, 28(117), 13-20. h ttp://dx.doi.org/10.15517/AP.V28I117.14131

Sapolsky, R. M. (1996). Why stress is bad for Your brain. Science, 273(5276), 749-750. https://doi.org/10.1126/science.273.5276.749

Savitz, J., Lucki, I., \& Drevets, W. C. W. (2009). 5-HT(1A) receptor function in major depressive disorder. Progress in Neurobiology, 88(1), 17-31. http s://doi.org/10.1016/j.pneurobio.2009.01.009

Szabo, S. T., \& Blier, P. (2001). Serotonin (1A) receptor ligands act on norepinephrine neuron firing through excitatory amino acidand gaba(A) receptors: A microiontophoretic study in the rat locus coeruleus. Synapse, 42(4), 203-212. https://doi.org/10.1002/syn.10009

Tovote, P., Fadok, J. P., \& Lüthi, A. (2015). Neuronal circuits for fear and anxiety. Nature Reviews, 16(6), 317-331.

Villalón, C. M., \& Centurión, D. (2007). Cardiovascular responses produced by 5-hydroxytriptamine: A pharmacological update on the receptors/mechanisms involved and therapeutic implications. NaunynSchmiedeberg's Archives of Pharmacology, 376(1-2), 45-63. https://doi.or $\mathrm{g} / 10.1007 / \mathrm{s} 00210-007-0179-1$

Wilson, F. A., \& Rolls, E. T. (1990). Learning and memory are reflected in the responses of reinforcemente-related neurons in the primate basal forebrain. Journal of Neuroscience, 10(4), 1254-1267. https://doi.org/10 .1523/JNEUROSCI.10-04-01254.1990 
Wu, J., Kramer, G. L., Kram, M., Steciuk, M., Crawford, I. L., \& Petty, F. (1999). Serotonin and learned helplessness: A regional study of 5-HT1A, 5-HT2A receptors and the serotonin transport site in rat brain. Journal of Psychiatric Research, 33(1), 17-22. https://doi.org/10.1016/S0022-39 56(98)00041-7

\section{Notas}

46. Banasr, M., \& Duman, R.S. (2008). Glial loss in the prefrontal cortex is sufficient to induce depressi- ve-like behaviors. Biological Psychiatry, 64(10),

475. Dwivedi, Y., Mondal, A. C., Payappagoudar, G. V., \& Rizavi, H. S. (2005). Differential regulation of serotonin (5-HT)2A receptor mRNA and protein levels after single and repeated stress in rat brain: Role in learned helplessness behavior. Neuropharmacology, 48(2), 204-214. doi:10.1016/j.neuropharm.2004.10.004 Evenden, J. (1999). Impulsivity: A discussion of clinical and experimental findings. Journal of Psychopharmacology, 13(2), 180-192. doi:10.1177/026988119901300211 Garfield, D. A., Fichtner, C. G., Leveroni, C., \& Mahableshwarkar, A. (2001). Open trial of ne- fazodone for combat veterans with posttrauma- tic stress disorder. Journal of Traumatic Stress, 14(3), 453-460. doi:10.1023/A:1011148304140 Gillin, J. C., SmithVaniz, A., Schnierow, B., Rapaport, M. H., Kelsoe, J., Raimo, E. M., Matthew, R., Goyette, L. M., Stein, M., \& Zisook, S. (2001). An open-label, 12-week clinical and sleep eeg study of nefazodone in chronic combat-related posttraumatic stress disorder. Journal of Clinical Psychiatry, 62(10), 789-796. doi:10.4088/JCP. v62n1007 Gould, E. (1999). Serotonin and hippocampal neurogenesis. Neuropsychopharmacology, 21, 46S-51S. Gould, E., \& Tanapat, P. (1999). Stress and hippocam- pal neurogenesis. Biological Psychiatry, 46(11), 1472-1479. doi:10.1016/S0006-3223(99)00247-4 Hariri, A. R., Mattay, V. S., Tessitore, A., Kolachana, B., Fera, F., Goldman, D., Egan, M. F., \& Weinberger, D. R. (2002). Serotonin transpor- ter genetic variation and the response of the human amygdala. Science, 297(5580), 400-403. doi:10.1126/science.1071829 Jauregui-Huerta, F., Ruvalcaba-Delgadillo, 